\title{
Green synthesis of silver nanoparticles using tannins
}

\author{
Pandian Bothi Raja ${ }^{1}$, Afidah Abdul Rahim ${ }^{1 *}$, Ahmad Kaleem Qureshi ${ }^{2}$, \\ KHALIJAH AWANG ${ }^{2}$ \\ ${ }^{1}$ School of Chemical Sciences, Universiti Sains Malaysia, 11800 USM, P. Pinang, Malaysia \\ ${ }^{2}$ Department of Chemistry, Faculty of Science, University of Malaya, 50603 Kuala Lumpur, Malaysia
}

\begin{abstract}
Colloidal silver nanoparticles were prepared by rapid green synthesis using different tannin sources as reducing agent viz. chestnut $(\mathrm{CN})$, mangrove $(\mathrm{MG})$ and quebracho $(\mathrm{QB})$. The aqueous silver ions when exposed to $\mathrm{CN}$, MG and QB tannins were reduced which resulted in formation of silver nanoparticles. The resultant silver nanoparticles were characterized using UV-Visible, X-ray diffraction (XRD), scanning electron microscopy (SEM/EDX), and transmission electron microscopy (TEM) techniques. Furthermore, the possible mechanism of nanoparticles synthesis was also derived using FT-IR analysis. Spectroscopy analysis revealed that the synthesized nanoparticles were within 30 to $75 \mathrm{~nm}$ in size, while XRD results showed that nanoparticles formed were crystalline with face centered cubic geometry.
\end{abstract}

Keywords: tannins; silver nanoparticles; green synthesis; XRD; SEM; TEM

(C) Wroclaw University of Technology.

\section{Introduction}

Nano structured noble metal/materials have an extensive range of applications in the fields of photonics [1, 2], microelectronics [3, 4], catalysis [5], biosensing [6] and antimicrobial functionalities [7], etc. Among the noble metals, silver nanoparticles are perhaps the most widely recognized for those applications. Their unique size-dependent physical chemical and biological properties make these materials superior and indispensable.

Physical and chemical procedures have been used for the synthesis of large quantities of nanoparticles in a relatively short time. A number of methods were used in the past decades for the synthesis of silver nanoparticles viz. reduction in solutions [8], radiation assisted synthesis [9] chemicaland photo-reduction in reverse micelles [10] and thermal decomposition of silver compounds. However, these techniques remain expensive and sometimes involve the use of hazardous chemicals [1113].

Increasing awareness towards hazardless procedure - green chemistry and other biological processes - have led to the development of an eco-

\footnotetext{
*E-mail: afidah@usm.my
}

friendly approach for the synthesis of noble metal nanoparticles [14]. This is achieved mostly by the use of plant sources viz. leaves, bark, fruit extracts [15, 16] and bioorganisms [17, 18]. The use of plant extract for the synthesis of nanoparticles could be advantageous over other environmentally benign biological processes by eliminating the elaborate process of maintaining cell cultures. These green methods are low cost, fast, efficient, and generally lead to the formation of crystalline nanoparticles with a variety of shapes and sizes between 1 and $100 \mathrm{~nm}$.

In continuation of these efforts, we report an eco-friendly, one step and ultra fast synthesis of silver nanoparticles by using silver salt $(\mathrm{AgCOOCH})_{3}$ and the natural reducing agents viz. $\mathrm{CN}, \mathrm{MG}$ and QB tannins. Furthermore, the synthesized silver nanoparticles have been duly characterized by UVVisible, FT-IR, XRD, SEM/EDX and TEM spectral techniques.

\section{Experimental}

\subsection{Materials}

Mangrove bark samples were collected from Matang forest, Malaysia and the tannin was prepared as reported elsewhere [19]. Chestnut tan- 
nin and quebracho tannins were purchased from SILVACHIMICA, Italy, while AR grade silver acetate $\left(\mathrm{AgCOOCH}_{3}\right)$ was purchased from Fischer Scientific Company (UK) and used without any further purification. The structures of hydrolyzable and condensed tannin are depicted in Fig. 1. Deionized water was used as the medium for the whole study; for example in preparation of tannin solutions $(100 \mathrm{mg} / \mathrm{L})$, silver acetate solution $(100 \mathrm{mg} / \mathrm{L})$, in synthesis of nanoparticles and dilution of nanoparticles.
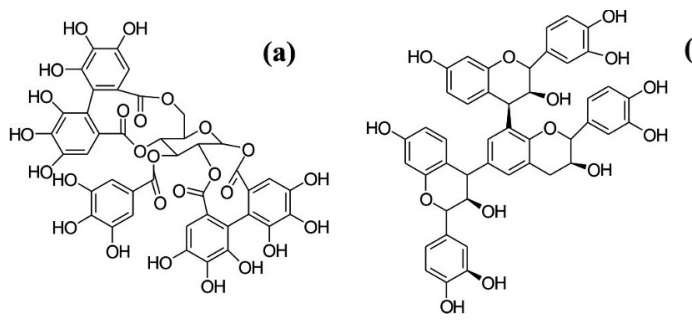

Fig. 1. Structure of (a) hydrolyzable tannin, (b) condensed tannin.

\subsection{Green synthesis of silver nano- particles}

An amount (10 mL) of $1000 \mathrm{ppm}$ concentrated silver acetate and tannins ( $\mathrm{CN}, \mathrm{MG}$ and $\mathrm{QB})$ was added separately and mixed well at room temperature $\left(30 \pm 2{ }^{\circ} \mathrm{C}\right)$ by using magnetic stirrer. The formation of silver nanoparticles was confirmed by the change in color intensity of the reaction mixture.

\subsection{UV-Visible absorption spectroscopy}

The bioreduction of silver acetate solution was periodically monitored with a UV-Visible spectrometer (Shimadzu, UV-2550, Japan). For the analysis, $0.2 \mathrm{~mL}$ of silver nanoparticles was taken in an optical path length quartz cuvette and was diluted to $2 \mathrm{~mL}$ using deionized water. The absorption maxima were measured at the wavelength range of 220 to $700 \mathrm{~nm}$.

\subsection{XRD analysis}

The X-ray diffraction (XRD) patterns were recorded using a PANalytical X'Pert PRO MRD PW3040. Samples for measurement were prepared by dropping $2 \mathrm{~mL}$ of silver colloids on silica plates and allowing them to dry at room temperature.

\subsection{SEM/EDX analysis}

The morphology of silver nanoparticles was examined using scanning electron microscopy (SEM) and energy dispersive X-ray spectroscopy (EDX) system (model: JSM-6460 LV). Silver nanoparticles were dispersed in deionized water under ultrasonic treatment, and then they were dropped on silica grid, dried and screened in SEM/EDX.

\subsection{TEM analysis}

Silver nanoparticles for transmission electron microscopy (TEM) observations were prepared by ultrasonically assisted dissolving the aqueous solution in double deionized water. A drop of the sample was subsequently dropped onto a lacey $\mathrm{C}$ film supported on a copper grid and then it was allowed to evaporate under ambient conditions. After drying, the nanoparticles were visualized using Phillips CM12 with Docu Version 3.2 transmission electron microscope.

\subsection{FT-IR spectroscopy}

Synthesized silver nanoparticles were then characterized by FT-IR spectroscopy using a Perkin Elmer System 2000 FT-IR instrument. For this purpose, synthesized silver nanoparticles were centrifuged at $4000 \mathrm{rpm}$ for 25 minutes to remove the free biomass or other components present in the solution. The centrifuged suspension was collected, re-dispersed in $10 \mathrm{~mL}$ sterile distilled water and the process was repeated for 3 times. Finally, the samples were dried and screened for FT-IR spectroscopy by using $\mathrm{KBr}$ pellet method.

\section{Results and discussion}

\subsection{Green synthesis and UV analysis}

In a typical synthesis, the color of the tannin solution changed immediately from yellowish brown to whitish green, light brown to dark brown, and brown to brownish black for $\mathrm{CN}, \mathrm{MG}$ and $\mathrm{QB}$, respectively, after reacting with $\mathrm{AgCOOCH}_{3}$. The change of color was an indication of the forma- 
tion of silver nanoparticles, and the final color of the solution varied with the amount of the starting materials.

The colloidal silver nanoparticles were then screened in UV-Visible spectroscopy, which is one of the most widely used techniques for structural characterization of silver nanoparticles. Fig. 2 show the UV-Visible absorption spectra of the colloidal silver nanoparticles as a function of time for $\mathrm{CN}$, MG, and QB tannins, respectively. An increase in absorbance with respect to time can be noticed in the UV-Vis spectrum (Fig. 2). Also, the peak area at 430 to $450 \mathrm{~nm}$ has increased with increasing reaction time for all the tannins studied. The sharp peak at around $435 \mathrm{~nm}$ evidences the formation of silver nanoparticles [20], while the increase in intensity could be due to increasing number of nanoparticles formed as a result of reduction of silver ions present in the aqueous solution [21].

\subsection{XRD studies}

The XRD patterns of tannin-silver nanoparticles (Fig. 3) show a sharp peak at $38^{\circ}$, which corresponds to (111) of the cubic silver structures within experimental error. Only one or two peaks can be observed because of the low concentration of silver nanoparticles in the product [22]. The broadening of Bragg's peaks indicates the formation of nanoparticles. Next, the mean size of silver nanoparticles was calculated using the DebyeScherrer's equation by determining the width of the (111) Bragg reflection [23]. The size of the synthesized nanoparticles was thus determined to be $57 \mathrm{~nm}$ for $\mathrm{CN}$, while $34 \mathrm{~nm}$ for $\mathrm{MG}$ and QB.

\subsection{SEM/EDX studies}

To attain further insight into the features of the silver nanoparticles, SEM and also the element analysis of the silver nanoparticles were performed using EDX on the SEM. SEM/EDX images of silver nanoparticles synthesized using $\mathrm{CN}, \mathrm{MG}$, and QB tannins, are shown in Fig. 4. The synthesized silver nanoparticles are well dispersed with minimum aggregation and possess different shapes, similar to the shapes obtained by TEM observations. The range of particle size was found to be

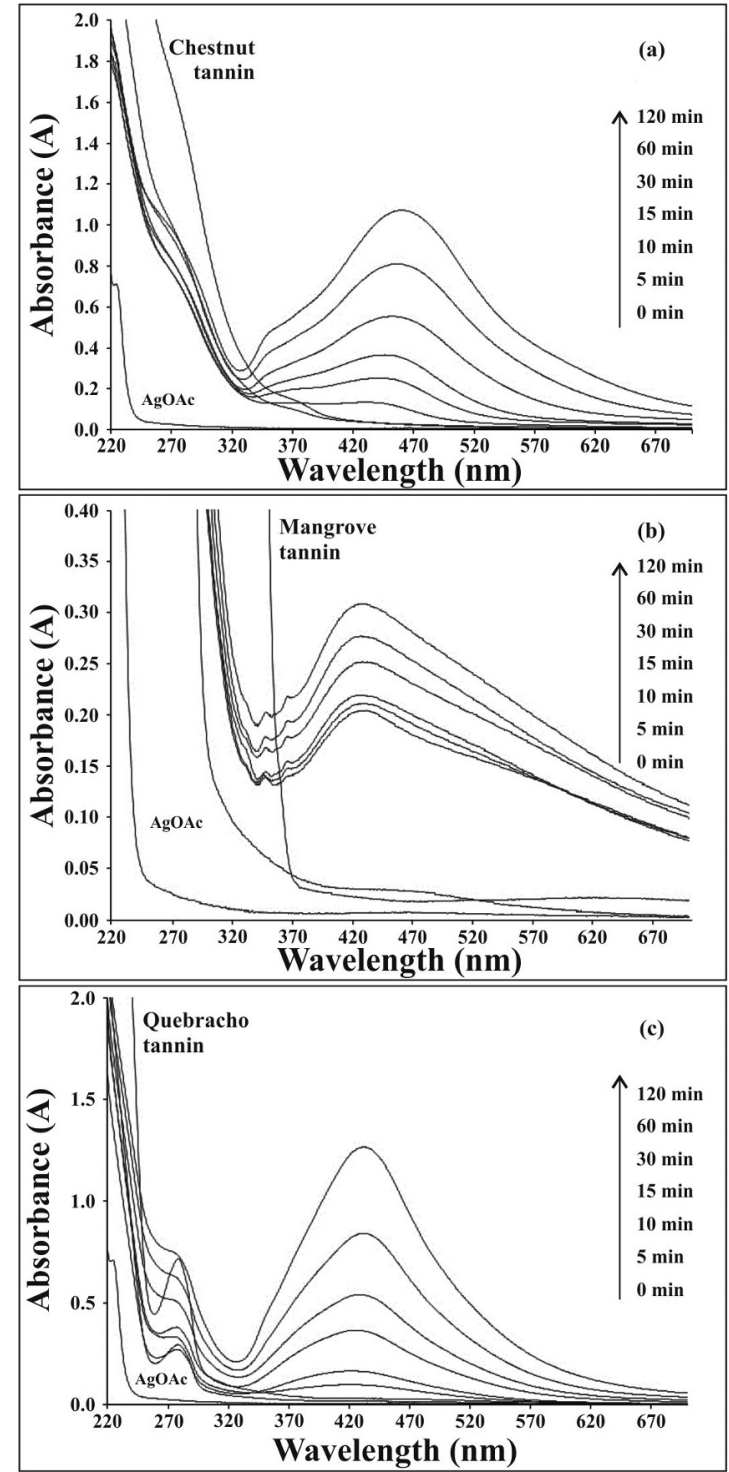

Fig. 2. UV-Visible spectra of (a) CN, (b) MG, and (c) QB tannin reduced silver nanoparticles at different time intervals.

larger for $\mathrm{CN}$ (59 to $69 \mathrm{~nm}$ ) as compared to $\mathrm{MG}$ and QB (35 to $45 \mathrm{~nm}$ ). EDX spectrum shows the typical absorption peak approximately at $3 \mathrm{keV}$ due to surface Plasmon resonance [24]. The presence of the elemental silver can be deduced from the EDX analysis, which also supports the XRD results. This indicates the reduction of silver ions to elemental silver. 


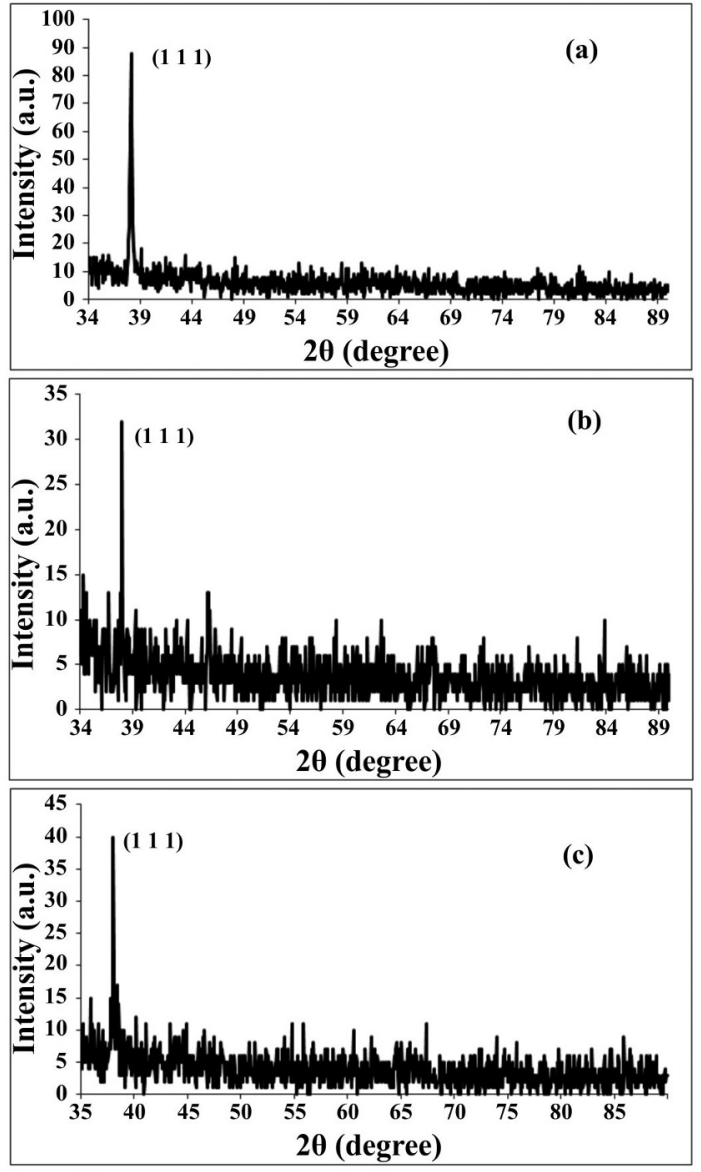

Fig. 3. XRD spectra of (a) $\mathrm{CN}$, (b) $\mathrm{MG}$, and (c) QB tannin reduced silver nanoparticles.

\subsection{TEM studies}

Fig. 5 shows the TEM images of the silver nanoparticles produced by $\mathrm{CN}, \mathrm{MG}$, and $\mathrm{QB}$, respectively. The silver nanoparticles have been formed in different shapes (circular, triangle, truncated triangle, ellipse, etc.) but most of them are circular in shape with smooth edges. Measurement of particles size has revealed that for $\mathrm{CN}$ it is in the range of 58 to $62 \mathrm{~nm}$, while for MG and QB in the range of 30 to $35 \mathrm{~nm}$. Furthermore, capping ability of tannins can also be observed from the images (Fig. 5).

\subsection{FT-IR spectroscopy analysis}

FT-IR spectroscopy measurements were carried out to identify the functional group changes on tan-

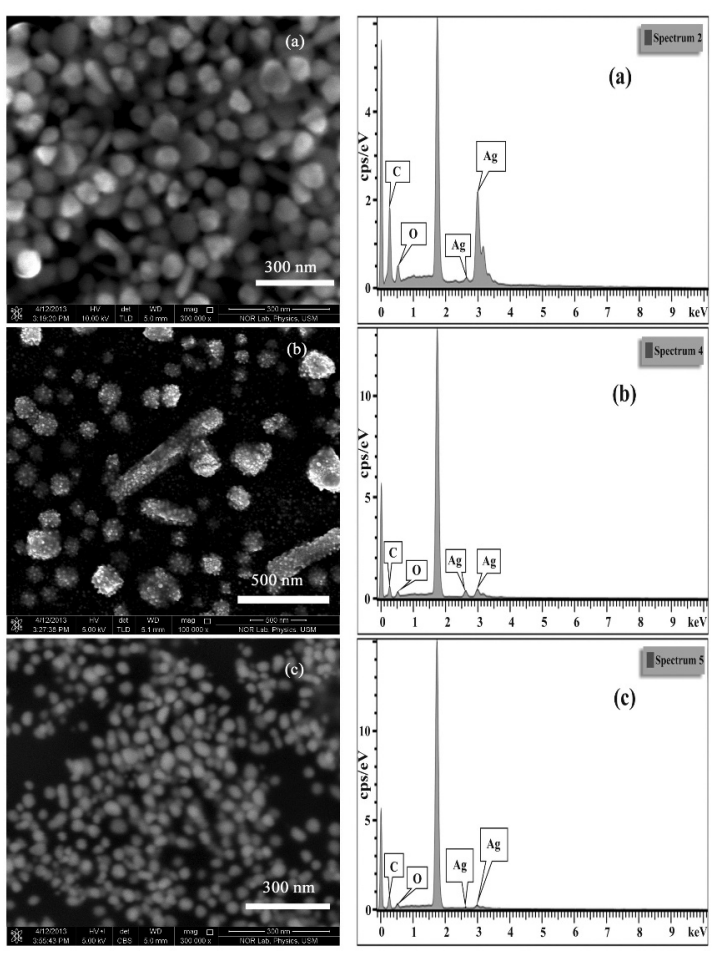

Fig. 4. SEM/EDX spectra of (a) CN, (b) MG, and (c) QB tannin reduced silver nanoparticles.

nin molecule when binding specifically on the silver surface. FT-IR spectra of CN, MG, and QB tannins, and their corresponding colloidal silver nanoparticles, are depicted in Fig. 6.

In Fig. 6, band (A) shows the presence of a broad absorption band around $3400 \mathrm{~cm}^{-1}$ for hydroxyl groups, three bands around 1610, 1494 and $1450 \mathrm{~cm}^{-1}$ as characteristic peaks for aromatic ring, and various peaks between 1000 and $1300 \mathrm{~cm}^{-1}$, corresponding to substituted benzene ring. Furthermore, it is also observed that FT-IR spectra of MG and QB show close resemblance to the spectra classified as condensed tannins [25], while that of $\mathrm{CN}$ show a strong absorption at $1734 \mathrm{~cm}^{-1}$ corresponding to the existence of $\mathrm{C}-$ $\mathrm{O}$ stretch of the ester group, which was classified as hydrolyzable tannin [26]. FT-IR spectra of silver nanoparticles - Fig. 6 (B) - show similar characteristic bands corresponding to their $\mathrm{CN}, \mathrm{MG}$, and QB tannins, which may be due to capping of tannins over silver metal. However, careful screening of Fig. 6 band (B) reveals broadening/weakening of 


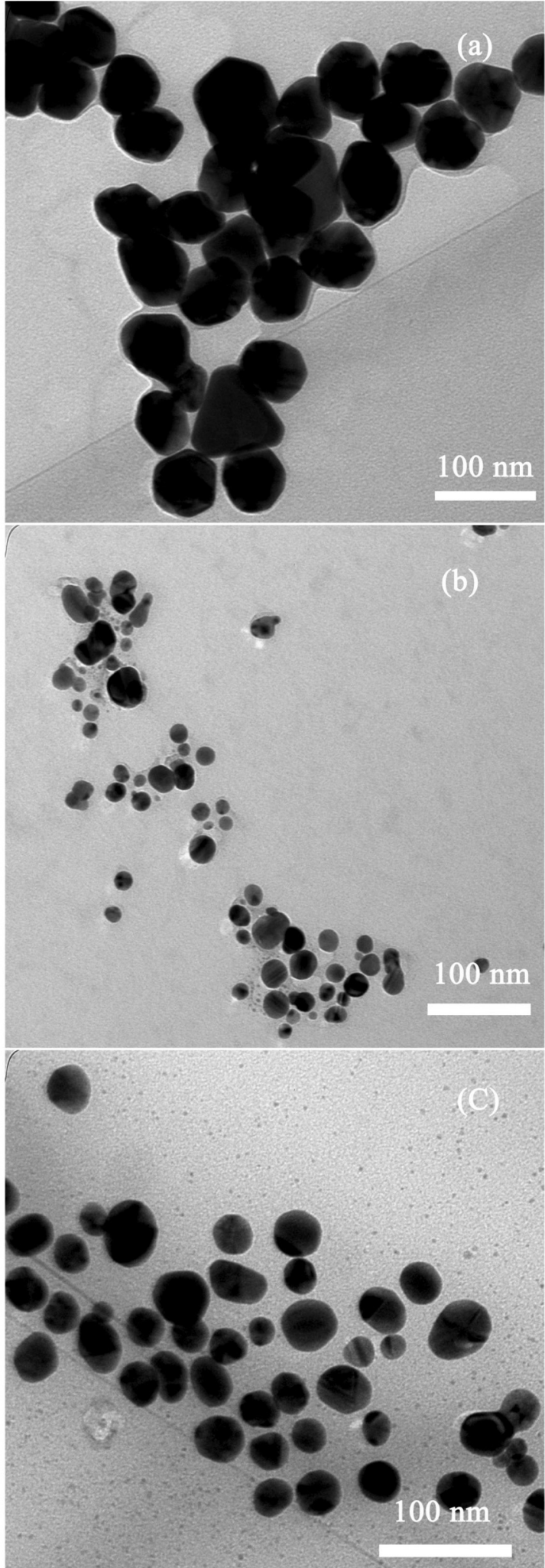

Fig. 5. TEM images of (a) $\mathrm{CN}$, (b) $\mathrm{MG}$, and (c) QB tannin reduced silver nanoparticles.
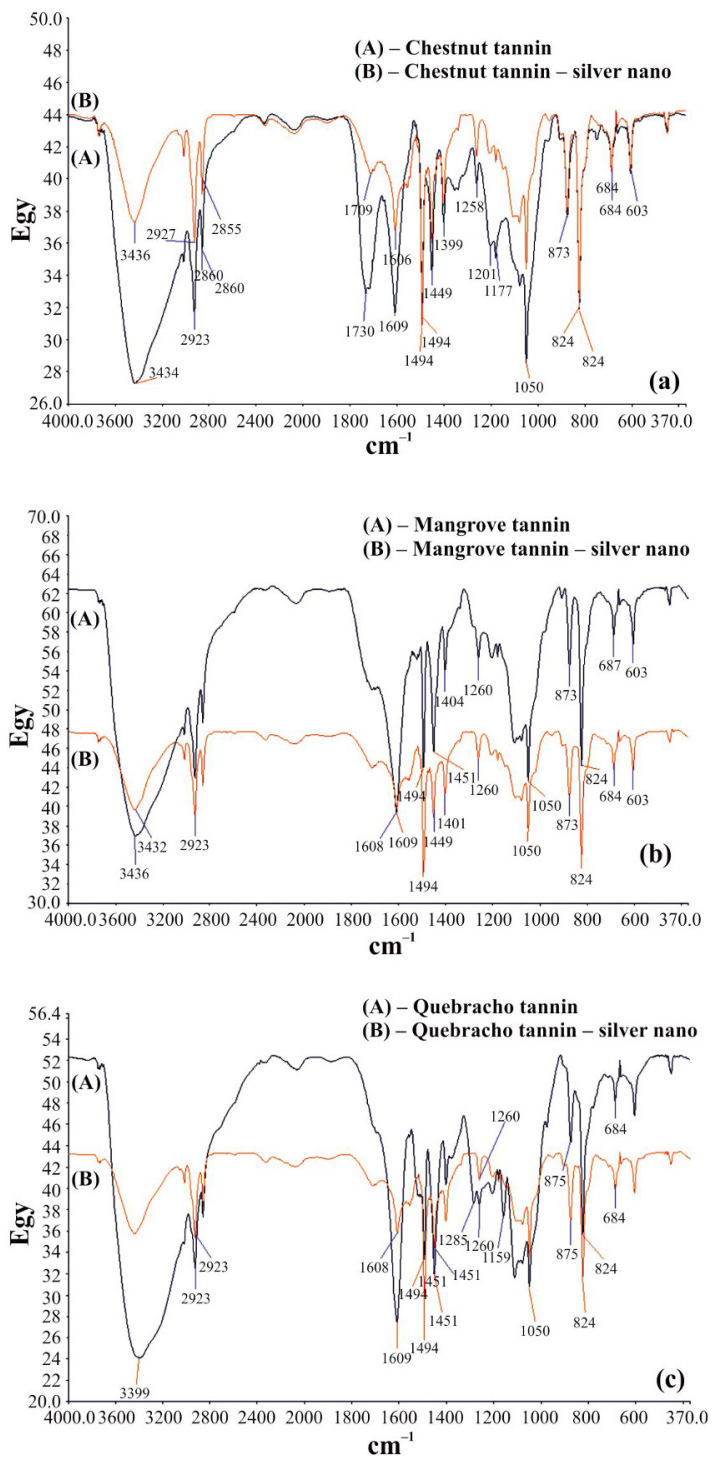

Fig. 6. FT-IR spectra of (a) CN, (b) MG, and (c) QB tannins (A) and reduced silver nanoparticles (B).

absorption bands for hydroxyl group $\left(3400 \mathrm{~cm}^{-1}\right)$ for all tannins studied; in particular $\mathrm{CN}$ (Fig. 6a) shows reduction of $\mathrm{C}-\mathrm{O}$ group band at $1734 \mathrm{~cm}^{-1}$, and QB (Fig. 6c) shows merging of $\mathrm{C}-\mathrm{O}-\mathrm{C}$ stretch peaks at $1284 \mathrm{~cm}^{-1}$.

These observations imply that all the tannins studied have been involved in reduction of silver ions to silver nanoparticles, mainly through hydroxyl groups. Specific reduction of $\mathrm{C}-\mathrm{O}$ group by $\mathrm{CN}$ tannin and $\mathrm{C}-\mathrm{O}-\mathrm{C}$ group by $\mathrm{QB}$ tannin has also been observed. Thus, FT-IR studies supported the 
capping ability of tannin over silver surface and evidenced the possible functional groups capable of reducing the silver ions.

\section{Conclusions}

A facile, green way was reported for the synthesis of silver nanoparticles using tannins as the reducing agent. The reduction of silver ions by tannins resulted in the formation of stable silver nanoparticles with spherical, triangle, truncated triangles, and decahedral morphologies of sizes ranging between 35 and $70 \mathrm{~nm}$. Tannins were able to reduce the silver nanoparticles by rapid rate of reaction and the resultant silver nanoparticles could have potential applications in optics, biomedical and biotechnological applications. Furthermore, this biosynthesis has several advantages viz. cost-effectiveness, compatibility for biomedical and pharmaceutical applications as well as for large-scale commercial production.

\section{Acknowledgements}

The authors gratefully acknowledge the financial support provided by Universiti Sains Malaysia (RU1001/PKMIA/811213) and University of Malaya Research Grants UMRG RG011/09BIO.

\section{References}

[1] Gould I.R., Lenhard J.R., Muenter A.A., Godleski S.A. FARID S., J. Am. Chem. Soc., 122 (2000), 11934.

[2] You C.C., Chompoosor A., Rotello V.M., Nano Today, 2 (2007), 34.

[3] Schimd G., Chem. Rev., 92 (1992), 1709.

[4] Deheer W.A., Rev. Mod. Phys., 65 (1993), 611.

[5] Kayanuma Y., Phys. Rev. B, 38 (1998), 9797.

[6] Chen J.Y., Wang D.L., Xi J.F., Au L., SiekKinen A., WARsen A., Li Z.Y., Zhang H., XiA Y., Li X., Nano Lett., 7 (2007) 1318.

[7] DU W.L., NiU S.S., XU Y.L., XU Z.R., FAN C.L., Carbohyd. Polym., 75 (2009), 385.
[8] Taleb C., Petit M., Pileni P., Chem. Mater. 9 (1997), 950.

[9] Henglein A., Langmuir, 17 (2001), 2329.

[10] Esumi K., Tano T., Torigoe K., Meguro K., Chem. Mater., 2 (1990), 564.

[11] Chen M., Wang L.Y., Han J.T., Zhang J.Y., Li Z.Y., QIan D.J., J. Phys. Chem. B, 110 (2006), 11224.

[12] Lou X.W., YuAn C.L., Archer L.A., Chem. Mater, 18 (2006), 3921.

[13] Kuo P.L., Chen W.F., J. Phys. Chem. B, 107 (2003), 11267.

[14] Hubenthal F., Noble Metal Nanoparticles: Synthesis and Optical Properties, in: ANDREWs D.L., SCHOLES G.D., WIEDERRECHT G.P. (Eds.), Comprehensive Nanoscience and Technology. Volume 1: Nanomaterials, Elsevier B.V., New York, 2011, p. 375.

[15] Jin E.S., Ghodake G.S., Deshrande N.G., LeE Y.P., Colloid. Surface. B, 75 (2010), 584.

[16] Sadowski Z., Maliszewska I.H., GrochowalSKA B., PolowczyK I., Koźlecki T., Mater. Sci.Poland, 26 (2008), 419.

[17] Gardea-Torresdey J.L., Gomez E., PeraltaVidea J., PARsons J.G., Troiani H.E., YaCAMAN M.J., Langmuir, 13 (2003), 1357.

[18] Sanghi R., Verma P., Bioresource Technol., 100 (2009), 501

[19] Shah A.M., Rahim A.A., Hamid S.A., Yahya.S., Raja P.B., Pigm. Resin. Technol., 40, (2011) 118.

[20] Huang J., Li Q., Sun D., LU Y., Su Y., Yang X., Wang H., Wang Y., Shao W., He N., Hong J., CHEN C., Nanotechnology, 18 (2007), 105104.

[21] KaViYa S., Santhanalakshmi J., Viswanathan B., Mater. Lett., 67 (2012), 64.

[22] Chen M., Wang L.Y., Han J.T., Zhang J.Y., Li Z.Y., QIAN D.J., J. Phys. Chem. B, 110 (2006), 11224.

[23] Borchert H., Shevchenko E.V., Mekis R.A., Kornowski A., Grubel G., Weller H., Langmuir, 21 (2005), 1931.

[24] Magudapathy P., Gangopadhyay P., Panigrahi B.K., NAIR K.G.M., Dhara S., Physica B, 299 (2001), 142.

[25] Pizzi A., Stephanou A.A., J. Appl. Polym. Sci., 50 (1993), 2105.

[26] Martinez S., Štagljar I., J. Mol. Struc.THEOCHEM, 640 (2003), 167.

Received 2014-04-09 Accepted 2014-05-13 